\title{
Rapid Mid-Infrared Variability in Protostellar Disks
}

\author{
T.T. Ke ${ }^{1}$, H. Huang ${ }^{1}$, D.N.C. Lin ${ }^{2,3}$
}

\begin{abstract}
Spectral energy distribution (SED) in protostellar disks is determined by the disks' internal dissipation and reprocessing of irradiation from their host stars. Around $\mathrm{T}$ Tauri stars, most mid-infrared (MIR) radiation (in a few to a few ten $\mu$ m wavelength range) emerge from regions around a fraction to a few AU's. This region is interesting because it contains both the habitable zone and the snow line. Recent observations reveal that SED variations, in the MIR wavelength range. These variations are puzzling because they occur on time scale (a few days) which is much shorter than the dynamical (months to years) time scale at $1 \mathrm{AU}$ to a few AU's. They are probably caused by shadows casted by inner onto outer disk regions. Interaction between disks and their misaligned magnetized host stars can lead to warped structure and periodic SED modulations. Rapid aperiodic SED variations may also be induced by observed X-ray flares from $\mathrm{T}$ Tauri stars. These flares can significantly modulate the ionization fraction of the gas and the net charge carried by the grains near the surface of the inner disk. The newly charged grains may be accelerated by the stellar or disk magnetic field and adjust their distances from the midplane. Shadows casted by these grains attenuates the flux of stellar photons irradiated onto regions at several AU's from the central stars. We use this model to account for the observed rapid aperiodic SED variabilities. We suggest regular monitoring of SED variations will not only provide valuable information on the distribution of the disk aspect ratio near the habitable zone but also provide a probe on the interaction between the inner regions of the disk with the magnetosphere of their host stars.
\end{abstract}

\section{Introduction}

For mid-infrared spectrum in protosteller disks, normally we expect that the spectral variation is determined by the local disk dynamics, with typical timescale comparable to the orbital period. Spectrum around a few $(<10) \mu m$ comes from disk irradiation at $0.1 \sim 1$ AU, with a typical dynamical timescale as $0.1 \sim 1$ year. However, a series of recent observations of $\mathrm{T}$ Tauri stars by Spitzer revealed the rapid variability of spectral energy distribution in protostellar disks with

\footnotetext{
${ }^{1}$ School of Physics, Peking University, Beijing 100871, China

${ }^{2}$ Kavli Institute for Astronomy \& Astrophysics, Peking University, Beijing 100871, China

${ }^{3}$ UCO/Lick Observatory, University of California, Santa Cruz, CA 95064, USA
} 
timescales as short as one week. Muzerolle et al. showed remarkable mid-infrared variability of the transitional disk LRLL 31 (Muzerolle et al. 2009), and highlighted the seesaw feature: "the variability observed at shorter wavelengths is reversed at longer wavelengths, with a constant pivot point at $\lambda \sim 8.5 \mu \mathrm{m}$." The largest flux difference reported is $60 \%$ at short wavelengths and $30 \%$ at long wavelengths, and an impressive change (20\%-30\%) occurred over just one week. Moreover, the flux variations are fairly constant as a function of wavelength within intervals $5-7 \mu \mathrm{m}$ and $11-35 \mu \mathrm{m}$, respectively. As a prevailing phenomenon, variability of disk emission has also been reported in premain sequence Herbig Ae stars (Sitko et al. 2008). Structural changes in the region of the disk near dust sublimation zone were proposed to account for the variability. For relatively slow variabilities, Bary et al. (2009) reported significant variations of $10 \mu \mathrm{m}$ silicate features on monthly and yearly timescales. They further pointed out several scenarios that might produce such variability, including intervening cooler dust grains, disk shadowing and illumination, turbulent mixing, and disk winds. Most recently, an extensive study (Flaherty et al. 2011) has been carried out for the transitional disk LRLL 31 with multiple epoches of observations. Based on the observations, authors reasonably put some constraints on the physical mechanism responsible for the variability based on their observations: "the variable accretion and wind are unlikely to be the cause of the variability, nor is a companion within $\sim 0.4 A U$ perturbing the disk." The most likely explanation they provided is "either a companion beyond $\sim 0.4 A U$ or a dynamic interface between the stellar magnetic field and the disk leading to a variable scale height and/or warping of the inner disk."

Following these puzzling observations, several mechanisms have been proposed to explain the rapid mid-infrared variability. Flaherty and Muzerolle considered non-axisymmetric structure variations in the inner disk (Flaherty and Muzerolle 2010), such as a warp or a spiral wave, and suggested the observed variability could be explained by a warped inner disk with variable scale height. As far as we know, this is the only paper trying to construct a physical model to explain the rapid mid-infrared variability at present. Though their model can generate the pivot point in the SED with a consistent timescale, their the location of the pivot point is inconsistent with the observations. Moreover, their model doesn't fit the flux change at $\lambda>8 \mu m$. Based on the CoRoT light-curves morphology of 83 previously known classical T Tauri stars, Alencar at al. associated the light curve variations with a magnetically controlled inner disk warp (Alencar et al. 2010). They further pointed out the inner warp dynamics should result from the interaction between the stellar magnetic field and the inner disk region. However, they haven't built up a physical model to consolidate their proposition, and their explanation concludes a timescale of a few rotational periods, which usually extends beyond one week, thus inconsistent with the observations. Espaillat et al. (2011) showed that they can reproduce the observed variability using irradiated disk models by changing the height of the inner disk wall by $\sim 20 \%$. Their model builts a reasonable link between the SED variability and the variation of the inner disk structure. However, they haven't provided a physical explanation about how inner disk wall can change in a short timescale. Based on a self-consistent physical model, we show in this paper, that observed X-ray flares can account for the rapid mid-infrared variability in protosteller disks. 
Stellar X-ray flares are prevailing among Young Stellar Objects (YSOs) (Feigelson and Montmerle 1999; Glassgold et al. 2005; Favata et al. 2005; Getman et al. 2008; Stelzer et al. 2005), which may come from material transfer from disk to the central star, or stellar magnetic reconnections. A recent COUP survey of the Orion Nebula Cluster (with Chandra space telescope) indicates that nearly all T Tauri stars emit X-ray $(0.5-8 \mathrm{keV})$ photons with a nearly constant quiescent flux in the range of $\sim 10^{23} \mathrm{~J} \mathrm{~s}^{-1}$. During COUP's two week observing period, 1-2 flares are commonly observed around each source(Wolk et al. 2005). These flares are characterized by power-law energy spectra and up to 1-2 orders of magnitude increase in their peak X-ray luminosity and they decay exponentially on a time scale $\sim 10^{3-5}$ s. Similar flares (some with larger amplitudes) were observed with the XEST survey of the Taurus region(Gudel and Telleschi 2007) and the Serpens star-forming region(Giardino et al. 2007). They have been modeled with emission from thermal plasma along cylinderical loops which extend beyond $R_{m}$ (Reale et al. 1997). These X-ray flares can greatly influence the circumstellar disks in various aspects. For example, (Wolk et al. 2005) estimated that the X-ray can ionize protostellar disks out to $1 \mathrm{AU}$ at a rate which is an order of magnitude higher than that estimated for cosmic rays. In this paper, we focus on the ionization effect of the X-ray flares.

We develop an analytical framework to show that the observed rapid X-ray flares from T Tauri stars can significantly modulate the ionization fraction of the gas and charges carried by the grains near the surface of the inner regions of their surrounding disks. In the proximity of their host stars, the newly charged grains are accelerated by the stellar and disk field and adjust their distances from the midplane. Consequently, the shadow casted by the grains on the surface of the inner disk regions attenuates in the flux of stellar photons irradiated at several AU's from the central stars. We use this model to account for the variabilities of spectral energy distribution, at a few micron range, on time scales of a few days.

The rest of this paper is organized as follows. Our primary objective is to identify and analyze the dominant physical process rather than to reproduce the SED evolution with the detailed construction of a new set of disk structure models. In Section 2, we briefly describe the static disk structure, mainly based on a model of Dullemond et al. (2001). We analyze X-ray ionization and grain charge loading process in the inner frontier of the disk in Section 3, and calculate the grain dynamics in Section 4. In Section 5, we formulate the spectrum variation, and discuss the response-time to verify the viability of our analysis. In Section 6, we present the main features of our model through a numerical example, with comparisons with observations. Finally, in section 7 , we conclude the paper and discuss different scopes in parameter space to further explore implications of our model.

\section{Disk Model}

In their seminal paper, Chiang and Goldreich (1997) proposed a hydrostatic radiative equilib-

rium model for passive disks surrounding $\mathrm{T}$ Tauri stars. The most notable idea they developed is a 
"sandwich" model for the vertical structure of the disk, which consists of an interior layer amidst two surface layers. The optically thin surface layer is directly heated by the stellar irradiation, and the interior region is heated by the re-irradiation from the surface layer. The direct implication of this model is the flaring configuration of the disk. Dullemond et al. (2001) modified this model by adding an inner hole due to the evaporation of carbon or silicon dusts in the disk. In their model, the inner rim of the disk is puffed-up, because of direct exposition to the stellar flux. As a consequence, there is region right beyond the inner rim which is in the shadow. Garaud and Lin (2006) further extended the disk structure by considering the both internal dissipation and surface irradiation.

In this section, we generally follow the line of Dullemond et al. (2001) with some modifications to incorporate internal dissipation, which dominates the energy spectrum in shadowed region. For sake of simplicity, we write down the main results of their works without detailed discussions. We consider a T Tauri star with a circumstellar disk, which is heated by both the irradiation of the central star and the internal viscous dissipation. Under the framework of the Minimum-Mass Solar Nebula Model, the surface mass density can be expressed in the form $\Sigma=\Sigma_{0}(R / A U)^{-3 / 2}$, with $\Sigma_{0}=10^{5} \mathrm{~kg} / \mathrm{m}^{2}$. The disk is divided into several regions: the puffed-up rim which is the inner edge of the disk locating in the sublimation line of silicates; the shadowed region which lacks the direct irradiation of the central star by the rim sheltering; the flaring part including the interior and surface layers. The intermediate shadowed region is heated by viscous dissipation whereas the outer part is mostly heated by stellar irradiation. For protostellar disks with a solar composition and accretion rate $\dot{M}_{d}$ around solar mass host stars, the transitional radius between these two regions is $r_{\text {tran }} \sim 1\left(\dot{M}_{d} / 3 \times 10^{-9} M_{\odot} y^{-1}\right)^{0.8}$ (Garaud and Lin 2006).

In most parts of the disk, small ( $\mu m$-size) grains are thought to be homogenously mixed with the gas, with the mass density percentage $Z=\frac{\rho_{\text {grain }}}{\rho_{\text {gas }}}=1 \%$. We take grains to be spheres with the average radius $r_{d}$ ranging from $0.1 \mu \mathrm{m}$ to $1 \mu \mathrm{m}$, and the density $\rho_{d} \sim 3 \times 10^{3} \mathrm{~kg} / \mathrm{m}^{3}$. The emissivity and correspondingly opacity possess two Gaussian peaks located at $10 \mu \mathrm{m}$ and $18 \mu \mathrm{m}$ due to silicate resonance. When dealing with the heat equilibrium equations, we omit the contribution of silicate resonance, and use $\varepsilon=T / T_{*}$ and $\kappa=\kappa_{v} T / T_{*}$ instead.

Optical height $H$ and temperature in the interior layer $T_{i}$ at radius $R$ can be expressed as

$$
\begin{aligned}
H & =\left(\frac{2}{7}\right)^{\frac{1}{7}} \chi^{\frac{8}{7}}\left(\frac{\psi_{s}}{2 \psi_{i}}\right)^{\frac{1}{7}}\left(\frac{T_{*}}{T_{0}}\right)^{\frac{4}{7}}\left(\frac{R}{R_{*}}\right)^{\frac{2}{7}} R, \\
T_{i} & =\left(\frac{2}{7}\right)^{\frac{1}{7}} \chi^{\frac{2}{7}}\left(\frac{\psi_{s}}{2 \psi_{i}}\right)^{\frac{2}{7}}\left(\frac{T_{*}}{T_{0}}\right)^{\frac{1}{7}}\left(\frac{R}{R_{*}}\right)^{-\frac{3}{7}} T_{*} .
\end{aligned}
$$

The dimensionless constant $\chi$ varies between 2 and 6 in the region discussed, which can be determined with an appropriate definition of the optical height (Garaud and Lin 2006). Functions $\psi_{i}$ and $\psi_{s}$ depend on the temperature in the interior layer and surface layer respectively, accounting for the possibility that the disk layer is not fully optically thick to its own emission (Dullemond et al. 2001). The symbols $T_{*}$ and $R_{*}$ represent, respectively, the surface temperature and radius of the central 
star. Constant $T_{0}=\frac{G M_{*} m_{p}}{k_{B} R_{*}}$ in unit of temperature, depends on stellar mass $M_{*}$ and radius $R_{*}$, and mass of hydrogen atom $m_{p}$.

Temperature and surface mass density for surface layer can be expressed as

$$
\begin{aligned}
T_{s} & =\left(\frac{R_{*}}{2 R}\right)^{\frac{2}{5}} T_{*}, \\
\Delta \Sigma & =\frac{\alpha\left(\frac{R_{*}}{R}\right)^{2} T_{*}^{4}}{2 \kappa_{p}\left(T_{s}\right) T_{s}^{4}},
\end{aligned}
$$

where $\alpha=\frac{2 H}{7 R}$ is the grazing angle of the irradiation from the central star to the surface of the flaring disk, and $\kappa_{p}\left(T_{s}\right)$ is the Planck mean opacity for surface layer.

Assuming the majority of dust grains in the disk as silicates, with evaporation temperature $T_{\text {evp }} \simeq 1500 \mathrm{~K}$, the disk will be truncated in a special radius $R_{\text {rim }}$, inside which, the grains are in the gaseous state mixed with gas of light elements and are optically thin to the stellar irradiation. In the case that self-irradiation cannot be ignored, the location and optical height of the rim can be jointly determined by following equations:

$$
\begin{aligned}
& R_{\text {rim }}=R_{*}\left(\frac{T_{*}}{T_{\text {evp }}}\right)^{2}\left(1+\frac{H_{\text {rim }}}{R_{\text {rim }}}\right)^{\frac{1}{2}}, \\
& H_{\text {rim }}=\chi_{\text {rim }}\left(\frac{T_{\text {evp }}}{T_{0}}\right)^{\frac{1}{2}}\left(\frac{R_{\text {rim }}}{R_{*}}\right)^{\frac{1}{2}} R_{\text {rim }},
\end{aligned}
$$

where the dimensionless constant $\chi_{\text {rim }}$ is different from that of the interior layer of the flaring disk because of the different grain density distribution and disk structures. From the discussion in Garaud and Lin (2006), one can expect that $\chi_{\text {rim }}$ will be some bigger than $\chi$, due to the optically thin inner hole.

The above expression for $R_{\text {rim }}$ is different from the truncation radius for the disk gas. Many $\mathrm{T}$ Tauri stars have strong (kG) fields on their surface(Johns-Krull et al. 2004; Johns-Krull 2007). These fields dominate the gas flow and truncation protostellar disks out to a distance

$$
R_{m}=\beta \mu^{4 / 7} \dot{M}_{d}^{-2 / 7}\left(2 G M_{*}\right)^{-1 / 7}
$$

where $\mu$ is the dipole magnetic moment, $\dot{M}_{d}$ is the accretion rate in the disk, and dimensionless parameter $\beta$ ranges from 0.5 to 1 (Konigl 1991). Since grains are the primarily opacity sources, the dust destruction radius is more relevant for radiation transfer and the location of $R_{\text {rim }}$ provided it is larger than $R_{m}$.

In most regions of the disk, especially at large radii, energy produced by the viscous dissipation is relatively small compared with stellar irradiation. For computational convenience, we consider the contribution of the frictional heat only in the shadowed region, stretching from the inner rim to the outer boundary $R_{f l}$. The following result further justifies this simplification. By equating 
$\frac{H_{\text {rim }}}{R_{\text {rim }}}$ with $\frac{H_{f l}}{R_{f l}}$ we obtain the locus of boundary at

$$
R_{f l}=\left(\frac{2}{7}\right)^{-\frac{1}{2}} \chi^{-4}\left(\frac{\psi_{s}}{2 \psi_{i}}\right)^{-\frac{1}{2}}\left(\frac{T_{*}}{T_{0}}\right)^{-2}\left(\frac{H_{\text {rim }}}{R_{\text {rim }}}\right)^{\frac{7}{2}} R_{*} .
$$

Following the framework developed by Ruden and Lin (Ruden and Lin 1986) on the basis of the $\alpha$-disk model (Shakura and Sunvaev 1973), we obtain the effective temperature $T_{e}$ in the shadowed region as

$$
T_{e}=\left(\frac{3 \sum_{0} \alpha_{s} k_{B}}{4 \sigma m_{p}}\right)^{\frac{1}{3}}\left(\frac{G M_{*}}{A U^{3}}\right)^{\frac{1}{6}}\left(\frac{R}{A U}\right)^{-1},
$$

where the dimensionless efficiency factor $\alpha_{s} \simeq 0.001$.

\section{X-Ray Ionization and Grain Charging}

Absorbed by grains and gas in the disk, the stellar X-ray can only penetrate a certain distance into the disk just like the visual photons do. Except for the directly exposed inner rim and the flaring surface layer, the structure of other regions in the disk is not significantly influenced by the $\mathrm{X}$-ray irradiation.

\subsection{Ionization state of the gas}

The rate of change in ionization fraction of the gas is

$$
\frac{\partial x}{\partial t}=(1-x)\left[\frac{\Gamma}{k_{B} T_{*}}+e(T) n x\right]-\left[\alpha_{B}(T) n x^{2}+\frac{Z \rho}{m_{d}} S_{e} v_{e} \sigma_{e}(Q) x\right]
$$

where $\Gamma$ is the ionizing energy input rate in $\mathrm{J} \mathrm{s}^{-1} \mathrm{H}$-atom ${ }^{-1}, T_{*}$ is the averaged source temperature, $\alpha_{B}(T)=3 \times 10^{-16} T^{-3 / 4}$ photons $\mathrm{m}^{3} \mathrm{~s}^{-1}$ is the case $\mathrm{B}$ recombination rate (Osterbrock and Ferland 2006), $e(T)=6 \times 10^{-17} T^{1 / 2} e^{-E_{0} / k_{B} T} \mathrm{~m}^{3} \mathrm{~s}^{-1}$ is collisional ionization rate (Drain 2011), and $n=\frac{\rho}{m_{p}}$ is the total number density of hydrogen. In the above equation, the last term takes into account the recombination of protons and electrons after they collide with the grains. $S_{e}, v_{e}$, and $\sigma_{e}$ are the absorption rate, velocity dispersion, and grains' cross section for electron capture which may be a function of the grains' net charge (see subsections below).

In the above equation, only photons that are more energetic than the ground state energy of the hydrogen atom, $E_{0}=13.6 \mathrm{eV}$ contribute to the flux of ionizing photons. The observed energy distribution(Hudson 1991; Crosby et al. 1993; Wolk et al. 2005) in the X-ray range suggests that $\mathrm{T}$ Tauri stars emit photon flux (ie photons per $m^{2}$ per second)

$$
\frac{d \dot{N}_{\gamma}(E)}{d E_{\gamma}}=\frac{\dot{N}_{0}}{E_{0}}\left(\frac{E}{E_{0}}\right)^{-1.7}
$$


where the normalization factor $\dot{N}_{0}$ can be obtained from the total X-ray luminosity $L_{X}$ in the energy range between $E_{0}$ and $E_{\max }$ (which is $8 \mathrm{kev}$ for the COUP sources),

$$
\frac{L_{X}}{4 \pi R_{\text {rim }}^{2}}=\int_{E_{0}}^{E_{\max }} E \frac{d \dot{N}_{\gamma}}{d E_{\gamma}} d E=\frac{\dot{N}_{0} E_{0}}{0.3}\left[\left(\frac{E_{\max }}{E_{0}}\right)^{0.3}-1\right] .
$$

In equation (10), the heating and ionization input rate

$$
\begin{gathered}
\Gamma=\int_{E_{0}}^{E_{\max }} E \sigma_{X}(E) \frac{d \dot{N}_{\gamma}(E)}{d E} d E \\
\frac{\Gamma}{k_{B} T_{*}}=\int_{E_{0}}^{E_{\max }} \sigma_{X}(E) \frac{d \dot{N}_{\gamma}(E)}{d E} d E
\end{gathered}
$$

where the X-ray photoionization cross section $\sigma_{X}(E)=\widetilde{\sigma}\left(E / E_{0}\right)^{-2.5}$, for photons' energy $E$, and $\widetilde{\sigma}=10^{-21} \mathrm{~m}^{2}$ (Glassgold et al. 1997). From equations (11), (12), and (14), we find

$$
\frac{\Gamma}{k T_{*}}=\frac{\dot{N}_{0} \widetilde{\sigma}}{3.2}\left[1-\left(\frac{E_{\max }}{E_{0}}\right)^{-3.2}\right] \simeq \frac{7.5 \times 10^{-3} L_{X} \widetilde{\sigma}}{R_{\text {rim }}^{2} E_{0}}\left(\frac{E_{\max }}{E_{0}}\right)^{-0.3} .
$$

This expression can be used to determine the changing rate of ionization fraction in equation (10).

The internal thermal energy $\left(E_{g}\right)$ equation of the gas can be written as (Lin and Murray 1992)

$$
m_{p}\left[\frac{d E_{g}}{d t}+p \frac{d(1 / \rho)}{d t}\right]=\Gamma(1-x)-n \Lambda(x, T)-n\left[\frac{3}{2} x^{2} k_{B} T \alpha_{B}(T)+x(1-x) e(T) E_{0}\right]
$$

where $p=\rho k T(1+x) / m_{H}$ is the pressure. At temperatures below $10^{4} \mathrm{~K}$, the cooling function $\Lambda(x, T)$ is given by Dalgarno \& McCray (1972) for optically thin line emission by metal ions with a solar abundance. Similar to equation (14), we replace $\Gamma$ with

$$
\Gamma=\frac{\dot{N}_{0} \widetilde{\sigma}}{2.2}\left[1-\left(\frac{E_{\max }}{E_{0}}\right)^{-2.2}\right] \simeq \frac{10^{-2} L_{x} \widetilde{\sigma}}{R_{\text {rim }}^{2}}\left(\frac{E_{\max }}{E_{0}}\right)^{-0.3} .
$$

In our model, the ionization fraction and the gas temperature of the gas are determined by the intensity of the ionizing photons emitted near the central star. During quiescence when the X-ray luminosity is less than $\sim 10^{23} \mathrm{~J} \mathrm{~s}^{-1}$, the surface of the disk region at $\sim 0.1$ AU retains its low gas temperature and ionization fraction. In this state, contribution from the collisional ionization rate $e(T)$ is negligible because free electrons and protons are more likely to collide with grains than directly with each other to recombine. With their relatively large cross section, grains easily can capture ionized electrons and protons, and become negatively charged.

However, during some outbursts when the flux of X-ray increases to $\sim 10^{25} \mathrm{~J} \mathrm{~s}^{-1}$. Nevertheless, $L_{X}$ is generally small compared with the stellar luminosity at longer wavelengths such that $T_{i}, T_{s}$, and $T_{e}$ are not affected by them. However, X-ray outbursts can significantly increase ionization fraction and the gas temperature, perhaps trigger the onset of thermal instability 
(Murray and Lin 1992) in the tenuous upper layers the disk at $\sim 0.1 \mathrm{AU}$. In this limit, collisions between the electrons and protons are important in the determination of the ionization fraction. Electrons' thermal energy is also elevated.

Now let's start estimating the equilibrium temperature and ionization fraction of the disk gas during quiescent state and X-ray flares. We show that equilibrium can indeed be reached within a relatively short time compared with the daily timescale of X-ray flare duration. By taking the right hand side of both equation (10) and (16) to be zero, we can solve the equilibrium temperature and ionization fraction as $T_{q} \simeq 472 K$ and $x_{q} \simeq 0.038$ in the quiescent state when the $\mathrm{X}$ ray luminosity $L_{X} \simeq 5 \times 10^{22} \mathrm{~J} / \mathrm{s}$. Note that the value of $T_{q}$ is small compared with $T_{i}$ produced by both viscous dissipation and reprocessed stellar photons in the visual wavelength range. Therefore the X-ray photons do not significantly modify the disk structure and ionization fraction during the quiescence.

However, the equilibrium temperature and ionization fraction as $T_{f} \simeq 9700 K$ and $x_{f} \simeq 0.79$ during the X-ray flares when the $\mathrm{X}$ ray luminosity increases to $L_{X} \simeq 1 \times 10^{25} \mathrm{~J} / \mathrm{s}$. In this phase, $T_{f}>T_{i}$ and photoionization by the $\mathrm{X}$-ray photons determines the thermal structure of the disk surface layer. In the above estimation, we are looking at gas/plasma with number density $n \simeq$ $10^{16} \mathrm{~m}^{-3}$ at 6 scale heights from the midplane $\left(\chi_{\text {rim }}=6\right)$ in the frontier of inner rim located around $0.1 A U$ from the central star (refer to the numerical section). The optical radial thickness of the inner rim is around $0.04 A U$ at 6 scale heights, which is comparable with $R_{\text {rim }} \sim 0.1 A U$ and $H_{\text {rim }} \sim 0.02 \mathrm{AU}$. Consistent with our previous intuition, both temperature and ionization get elevated during X-ray flares.

For dynamics of gaseous ionization by X-ray flares, we find the ionization fraction goes as $\left(1-e^{-t / \tau_{i}}\right)$ if we neglect the collisional ionization and direct recombination, with the response time

$$
\tau_{I}=\left(\frac{\Gamma}{k_{B} T_{*}}+\frac{Z \rho_{\text {rim }}}{m_{d}} S_{e} v_{e} \sigma_{e}\right)^{-1} \sim 10^{2} s
$$

which is much shorter than one day. By taking into account the collisional ionization and direct recombination, the ionization should proceed even faster. For heating and cooling dynamics, we estimate the response time respectively from the equation (16) is of the order

$$
\begin{aligned}
\tau_{H} & =\frac{k_{B}\left(T_{f}-T_{q}\right)}{\Gamma} \sim 1 s \\
\tau_{C} & =\frac{k_{B}\left(T_{f}-T_{q}\right)}{n \Lambda\left(T_{f}, x_{f}\right)} \sim 10 s
\end{aligned}
$$

which are again much shorter than the daily X-ray flare timescale. Finally we show that blackbody irradiation for grains is so effective that the conductive heat transfer from overheated ambient gas to grains is negligible. In fact, during X-ray flares, grains are heated not only by stellar flux, but also by the collisions with overheated gas. Thus instead of equation (5), we have

$$
\sigma T_{\text {grain }}^{4}=\sigma\left(\frac{R_{*}}{R_{\text {rim }}}\right)^{2} T_{*}^{4}\left(1+\frac{H_{\text {rim }}}{R_{\text {rim }}}\right)+6 n v_{H} k_{B}\left(T_{f}-T_{\text {grain }}\right)
$$


where $\sigma$ is the Stefan-Boltzmann constant, and average velocity of hydrogen atoms is approximated

as $v_{H}=\sqrt{\frac{3 k_{B} T_{f}}{m_{p}}}$. For ambient gas/plasma temperature during X-ray flares $T_{f} \simeq 9700 K$, the equilibrium temperature for grains $T_{\text {grain }}=1500 \mathrm{~K}$ which is comparable to the equilibrium temperature $T_{e v p}=1500 \mathrm{~K}$ in the quiescent state. Thus conductive heat transfer from ambient gas is entirely negligible compared with the stellar irradiation in determining temperature of grains in the inner rim. Last but not least, after the X-ray flare, the rise in the gas temperature implies that the disk scale height will increase by a factor of two on the local dynamical time scale (probably a few days), which will further lower the local gas density. As a result, the equilibrium temperature should be higher, and more charge will be loaded on grains. Thus grains experience a stronger magnetic force and a smaller gas drag, but at the same time a outward gas push. It's complicated to judge which effect dominates, and we suspect all to be insignificant. So in this paper, we omit the influence of the change of gas scale height right after X-ray flares.

\subsection{Charge-loading process}

We now consider the charge carried by the grains. X-ray flares charge grains mainly through two mechanism: collisional charging and photoelectric emission (Drain 2011). Photoelectric emission happens when a X-ray photon excites an energetic electron out of the grain surface. However the effect of photoelectric emission is comparable with collisional charging only when the photoelectric yield is of order unity. Hindered by multiple complications, photoelectric yield is estimated around 0.1 for the grain size and photon energy we considered (Weingartner et al. 2006), thus negligible. We omit the photoelectric emission and focus on the collisional charging effect below.

In the framework of a certain grain with charge $Q$, the cross sections for electrons and protons to collide with this grain are (Umebayashi 1983):

$$
\begin{aligned}
\sigma_{e} & =\pi r_{d}^{2} e^{\frac{1}{4 \pi \varepsilon_{0}} \frac{e Q}{r_{d} E_{e}}} \\
\sigma_{p} & =\pi r_{d}^{2}\left(1-\frac{1}{4 \pi \varepsilon_{0}} \frac{e Q}{r_{d} E_{p}}\right),
\end{aligned}
$$

where $e$ is the elementary electric charge, $\varepsilon_{0}$ is vacuum permittivity, $E_{e}=\frac{1}{2} m_{e} v_{e}^{2}$ and $E_{p}=\frac{1}{2} m_{p} v_{p}^{2}$ are the kinetic energy of protons and electrons respectively. As shown above, the temperature equilibrium can be achieved in a relatively short time, so that the energy should be equally partitioned between electrons and protons $E_{e} \simeq E_{p}=\frac{3}{2} k_{B} T_{f}$.

By introducing $S(e)$ and $S_{p}$ as the absorption rate for the electrons and protons after their collision with grains. We write the microscopic charge balance equation for grains:

$$
S_{e} n_{e} v_{e} \sigma_{e}=S_{p} n_{p} v_{p} \sigma_{p}
$$

where $n_{e}$ and $n_{p}$ are number density of protons and electrons respectively. By defining dimensionless parameter

$$
\theta=-\frac{1}{4 \pi \varepsilon_{0}} \frac{Q e}{r_{d} E_{e}}
$$


we can rewrite (24) as

$$
\frac{e^{-\theta}}{1+\theta}=\frac{n_{p}}{n_{e}} \frac{S_{p}}{S_{e}}\left(\frac{m_{e}}{m_{p}}\right)^{\frac{1}{2}}
$$

High speed electrons tend to have a lower absorption rate than that for protons, and we estimate

$0.01 \lesssim \frac{S_{e}}{S_{p}} \leq 1$ (Umebayashi 1983). Moreover as inferred from ionization fraction $x_{f} \simeq 0.79$, during X-ray flares, the electrons and protons are so abundant that the charge on grains consist of only a tiny portion of the entire plasma, thus we have $n_{e}=n_{p}$. With these restrictions, we find from equation (26) $2.5 \lesssim \theta \lesssim 6.4$, which is entirely determined by the ratio of absorption rates for electrons and protons. We adopt $\theta=5$ (with $S_{e}=0.05$ ) in the following analysis. Conclusively we know in the equilibrium state, grains are negatively charged to

$$
Q_{0}=-5 \frac{4 \pi \varepsilon_{0} r_{d} E_{e}}{e}
$$

which is proportional to the radius of the grain $r_{d}$ and the electrons' energy $E_{e}$, but independent of the ionization fraction and electron density. During X-ray flares, plasma temperature in the inner rim increases dramatically, leading to a increase in $E_{e}$ from $\frac{3}{2} k_{B} T_{q}$ to $\frac{3}{2} k_{B} T_{f}$, and eventually a propositional increase in $Q_{0}$ by $\frac{T_{f}}{T_{q}} \sim 20$ times.

Dynamics of charge loading processes can be determined by the following differential equation

$$
\frac{d Q}{d t}=-e S_{e} v_{e} \sigma_{e}(Q) n_{e}+e S_{p} v_{p} \sigma_{p}(Q) n_{p}
$$

We can determine the response time for charge loading on grains to be

$$
\tau_{Q} \sim \frac{\varepsilon_{0} E_{0}}{e^{2} n_{e} S_{e} v_{e} r_{d}} \sim 10^{-7} s
$$

which is very swift, thus it takes nearly no time for the grains to get charged.

In conclusion, we show in this section that X-ray flares will not only significantly ionize gas in the inner rim of the circumstellar disk, but also elevate its temperature by thousands of Kelvin higher. Both ionization and temperature elevation will accomplish in a much short timescale compared with X-ray flare duration. The overheated gas and plasma are shown to have negligible effect on equilibrium temperature of the grains, but will significantly increase charge on the grains in a relatively short time. In next section, we will show that the newly charged grains will undergo a collective migration driven by stellar magnetic field, thus modulate the height of inner rim of the disk.

\section{Grain Dynamics}

When the temperature in the inner rim is high enough $\left(\sim 10^{3} K\right)$ for collisional ionization of metal atoms, magnetic field can be coupled effectively with the gas in the inner region of a circumstellar accretion disk. However despite of voluminous studies on the interaction of stellar magnetic 
field and the circumstellar disk (Pringle and Rees 1972; Ghosh and Lamb 1979a; 1979b; Aly 1980; Konigl 1991; Livio and Pringle 1992; Shu et al. 1994; Havashi et al. 1996), there is little consensus on how well the plasma couple with the field lines. Some outstanding issues include "whether the disk excludes the stellar magnetic field by diamagnetic currents or the field can penetrate and thread a large fraction of the disk, whether the threaded field remains closed (connecting the star and the disk) or becomes open by differential shearing (Lai 1999)." There has also been a recent trend on 3D-modeling magnetic stellar field (Gregory et al. 2007; Jardine et al. 2007), which is very complicated and beyond the scope of our analytical framework. In this section, we first develop a parametric framework for the magnetic field, taking into account the interactions between the stellar field and the circumstellar disk; and then discuss the charged grain dynamics driven by the magnetic field.

As mentioned above, the circumstellar disk would be disrupted at the magenetospheric boundary $R_{m}$ where the magnetic stress is large enough to remove the excess angular momentum of the nearly Keplerian flow over a narrow transition zone and channel the plasma onto the stellar polar caps. The dynamic consequence of disk accretion onto the central star is to synchronize the stellar spin with the disk rotation. How much time it takes to reach an equilibrium state depends on the coupling intensity of field line and the disk. The corotational radius $R_{c o} \sim R_{m}$, and Konigl adopted $R_{c o}=2 R_{m}$ (Konigl 1991), and in fact the ratio can be even smaller. Within the range from $R_{c o}$ to $R_{m}$ the disk angular velocity departs significantly from the Keplerian value, and corotates with the central star; while beyond $R_{c o}$, the angular velocity is Keplerian (Ghosh and Lamb 1979a; 1979b). For a typical T Tauri stars, the spin period is around 8 days, so that $R_{c o} \sim 0.07 A U$, which as shown later, is smaller than $R_{\text {rim }}$ the sublimation frontier of the disk. In our parametric framework, we denote

$$
R_{c o}=\epsilon R_{\text {rim }},
$$

where the dimensionless parameter $\epsilon$ ranges from 0.3 to less than 1 . In order for the stellar magnetic field to induce a spin equilibrium (in which there is little net angular momentum flux between the stellar spin and the disk), it must be well coupled to the disk beyond the corotation radius (Ju et al in preparation).

We assume the stellar field can influence the surface layer of the disk near $R_{\text {rim }}$. We consider the case that stellar magnetic dipole, stellar spin and the disk angular momentum are aligned with each other. In the disk mid-plane, the magnetic field in the cylindrical coordinate can be expressed as:

$$
\begin{aligned}
B_{z} & =-B_{s} \frac{R_{*}^{3}}{R^{3}} \\
B_{\varphi} & =\mp \zeta B_{s} \frac{R_{*}^{3}}{R^{3}} \\
B_{R} & =0
\end{aligned}
$$

where $B_{s} \sim 10^{3} G s$ represents the magnetic strength at the equator at the surface of the central star. The negative sign for the longitudinal component $B_{z}$ comes from our consideration that 
the magnetic moment is parallel to the stellar spin. We leave the alternative anti-parallel case in the below discussion section. The azimuth component $B_{\varphi}$ comes from twisting of $B_{z}$, and the upper (lower) sign corresponds to the value at the upper (lower) disk surface. The quantity $\zeta$ specifies the azimuthal pitch of the field line. In general we expect $\zeta \lesssim 1$, but its actual value or form depends on details of the dissipative processes involved in the disk-magnetic field interactions. If the stellar magnetic field threads the disk in a closed configuration, we expect $\zeta \propto\left(\omega_{s}-\omega_{d}\right)$ (Livio and Pringle 1992), where $\omega_{s}$ and $\omega_{d}$ are angular momentum for central star and disk respectively. However, it has been argued that the differential shearing and the plasma flowing from the disk into the overlying magnetosphere will blow the field lines open and maintain them in an open configuration, in which case we expect $\zeta$ to be positive and of order unity (Lai 1999; Havashi et al. 1996). The radial component $B_{R}$ comes from the inward accretion flow with velocity $v_{R} \sim \nu / R$ which is much smaller than the azimuthal disk velocity, so we adopt $B_{R}=0$ in the analysis. Because $\frac{H_{\text {rim }}}{R_{\text {rim }}} \ll 1$ as shown in the following numerical example, when we discuss the grain dynamics, we can always approximate magnetic field as

$$
\mathbf{B}=\mathbf{B}_{D} \mp \zeta B_{s} \frac{R_{*}^{3}}{R^{3}} \hat{\varphi}
$$

in the proximity of disk mid-plane, where $\mathbf{B}_{D}$ is the magnetic field by unperturbed stellar dipole.

After grains in the inner rim get charged by X-ray ionization, they move upward (downward in antiparallel case) collectively driven by the magnetic field. As a consequence, both the shape and the location of the inner rim changes over time, with the scale of movement as the Lorentz radius $R_{L}=\frac{u m_{d}}{B_{D} Q_{0}}$, and the timescale as the Lorentz period $T_{L}=\frac{2 \pi m_{d}}{B_{D} Q_{0}}$. We focus on the dynamics of grains which originally settle around the upper/lower brim of the inner rim, and try to infer the variability of $H_{\text {rim }}$ based on these grains' migration.

The equation of motion for a certain grain in the rotating framework of central star can be formulated as

$$
m_{d} \dot{\mathbf{v}}_{r}=-m_{d} \frac{G M_{*} \mathbf{R}}{r^{3}}+Q_{0} \mathbf{v}_{\mathbf{r}} \times \mathbf{B}-\nu\left|\mathbf{v}-\mathbf{v}_{\mathbf{K}}\right|\left(\mathbf{v}-\mathbf{v}_{\mathbf{K}}\right)-2 m_{d} \omega_{\mathbf{s}} \times \mathbf{v}_{\mathbf{r}}-m_{d} \omega_{\mathbf{s}} \times\left(\omega_{\mathbf{s}} \times \mathbf{r}\right),
$$

where $\omega_{\mathbf{s}}$ is the spin angular velocity for central star, $\mathbf{v}_{\mathbf{s}}=\omega_{\mathbf{s}} \times \mathbf{R}$ is the corotation velocity with the central star, $\mathbf{v}_{\mathbf{r}}=\mathbf{v}-\mathbf{v}_{\mathbf{s}}$ is grain's velocity in the rotating framework, and $\mathbf{v}_{\mathbf{K}}$ is the Keplerian velocity. The last two terms are Coriolis and centrifugal force in the rotating framework. We only preserve the radial component of gravity, because the vertical component has been balanced with the gas turbulence, and that's the reason why the grain can stay around the brim initially. We neglect collisions between grains. Gas drag coefficient $\nu=\frac{1}{8} \pi r_{d}^{2} \rho$ is calculated based on the fact that the velocity of the grain is much larger than the thermal velocity of the gas, so the gas can be treated as still relative to the fast grains. Hermite scheme (Aarseth 2003) can be adopted to numerically solve the differential equation, with constant time steps much smaller compared with the Lorentz period. Notice that the Lorentz force decreases as $R^{-\frac{7}{2}}$, while the gravity decreases as $R^{-2}$. For the grains in the surface layer of the flaring disk around $R \gtrsim 3 A U$, the relative strength of the Lorentz force with respect to gravity becomes $\sim 10^{-3}$ of the value at the inner rim. Thus 
we can omit the magnetically driven dynamics of grains in the surface layer of the flaring region. After we get the grain dynamics, we approximate the variability of $H_{\text {rim }}$ as the location variations of grains in the brim.

\section{Spectrum Variability}

The collective outward migration of grains (in the parallel case) puffs up inner rim of the disk. Consequentially, the irradiation flux from the inner rim increases; while larger region in the flaring disk becomes shadowed by the rim. We track the flux variability of both inner rim and shadowed region, by tracking the variability of the height of inner rim $H_{\text {rim }}$. As shown below, the blackbody irradiation for individual grains is so effective that it takes almost no time for the newly shadowed (exposed) grains to reach a new equilibrium temperature. So we can determine the boundary $R_{f l}$ dividing the shadowed region and the exposed region by enabling geometric relationship instantly, the same as the static case (8):

$$
\frac{H\left(R_{f l}\right)}{R_{f l}}=\frac{H\left(R_{\text {rim }}\right)}{R_{\text {rim }}}
$$

Once the variability of disk structure determined, computation of the spectra before and after onset of X-ray flares is pretty standard (Dullemond et al. 2001).

Finally we justify the temperature response time for newly exposed/shadowed grains in the flaring part. For newly exposed grains in the surface layer, the temperature dynamics follows

$$
\pi r_{d}^{2} \sigma T_{*}^{4}\left(\frac{R_{*}}{R}\right)^{2}-4 \pi r_{d}^{2} \varepsilon \sigma T_{s}^{4}=\frac{4}{3} \pi r_{d}^{3} \rho_{d} c_{d} \frac{d T_{s}}{d t} .
$$

For grains around $3 A U$, it's not hard to figure out the temperature response time is around $10^{-3}$ day, which is much shorter than the spectrum variability with daily timescales as we are interested in. Similarly, the newly shadowed grains in the surface layer undergoes a rapid drop of temperature with timescales around $1 s$, much shorter than the daily timescale. As the irradiation from the surface layer can only penetrate into a distance in the interior layer, the newly exposed surface layer will heat a thin top interior layer quickly by the irradiation, without changing the temperature of the inner interior layer much. The surface mass density of quickly heated top interior layer $\Delta \Sigma_{i}=\rho_{i} l=\frac{\tau_{v}}{\kappa_{v}} \sim \frac{1}{\kappa_{v}}$, then the temperature dynamics follows

$$
\psi_{s} \alpha \sigma T_{*}^{4}\left(\frac{R_{*}}{R}\right)^{2}-2 \psi_{i} \sigma T_{i}^{4}=\frac{1}{\kappa_{v}}\left(\frac{C_{v}}{\mu_{H}}+Z c_{d}\right) \frac{d T_{i}}{d t},
$$

where $C_{v}=\frac{3}{2} R_{\text {gas }}$ is the gas specific heat per mol. We estimate the temperature response timescale for grains in the interior layer around $3 A U$ around $10^{2}$ days, which is much longer than the daily timescale we are interested in. In the above discussion, we omit the effect of the vertically convec-

tive heat transference. Since it has the time scale comparable to the local dynamics, the effect of convective heat transference should works at at timescale much longer than several days. Conclusively, the newly exposed/shadowed grains in the surface layer change instantly; while grains in the interior layer change little in the daily timescale. 


\section{Numerical Studies}

In this section, we apply our model to an numerical example to generate predictions and compare with observations. We take the central star as a spherical blackbody with $M_{*}=1.8 M_{\odot}$ (Muzerolle et al. 2009), $R_{*}=3 R_{\odot}, T_{*}=4000 \mathrm{~K}$. The outer cutoff radius of the disk $R_{\text {out }}=$ $2.3 \times 10^{2} A U$ (Chiang and Goldreich 1997). The radius of grains $r_{d}=0.5 \mu \mathrm{m}$. The value of $\chi$ takes different in different regions, where $\chi=4$ for the flaring disk and $\chi_{\text {rim }}=6$ for the inner rim due to a higher gas density (Dullemond et al. 2001; Dullemond and Monnier 2010; Garaud and Lin 2006). The stellar magnetic field at surface $B_{s}=1000 G s$, the azimuthal component coefficient in equation (32) $\zeta=0.5$ and dimensionless coefficient in equation (30) $\epsilon=0.5$.

We first describe the whole physical processes of spectral variability triggered by X-ray flares. We assume stellar X-ray flare with luminosity $\sim 10^{25} \mathrm{~J} / \mathrm{s}$ starts at time $t=0$, and lasts for one day. During X-ray outburst, gas in the inner rim gets ionized quickly, with the ionization fraction rising from around $4 \%$ to around $80 \%$ within $10^{2}$ seconds. Meanwhile the X-ray photons lead to a temperture enhancement of the gas in the disk surface layer from around $400 K$ to around $10^{4} K$ within the same timescale. As a result, speed of electrons become higher, and the collisions between electrons and grains become more frequently. In the equilibrium state, which can be reached in almost no time, the charge on grains increases by around 20 times. Grains strongly negatively charged will be driven by the magnetic field to undergo collective migration inward, in the case that the moment of magnetic dipole is parallel with the disk angular momentum. Consequently, the inner rim of the disk gets squeezed, and the shadow casted by the grains on the flaring disk attenuates. As a result, the spectrum at shorter wavelength increases while the spectrum at longer wavelength decreases, as sketched in Fig. 1. In our numerical example, the height of inner rim decreases by $17.6 \%$ in the first day, and decreases by $22.7 \%$ cumulatively in seven days after occurrence X-ray flares, as shown in Fig. 2. In fact as discussed before, location of the boundary of the shadowed region depends on the ratio of height over radial distance of the inner rim, we show variability of the height-radius ratio in Fig. 3. In Fig. 4, we plot the trajectory of charged grains projected in the midplane. We find newly charged grains still undergo circular orbit, with a minor inward shift. Finally in Fig. 5, we show the variability reproduced by our model. A clear "seesaw" feature is shown here: the spectrum at shorter wavelength decreases and spectrum at longer wavelength increases over time, with a pivot around $8 \mu \mathrm{m}$. Spectrum at $\lambda=5 \mu \mathrm{m}$ decreases by $14.9 \%$ and $18.7 \%$ respectively within one day and the whole week; while spectrum at $\lambda=15 \mu \mathrm{m}$ increases by $7.4 \%$ and $9.2 \%$ respectively within one day and the whole week. The result is consistent with observations described in the first section.

\section{Summary and Discussion}

In this section, we briefly discuss the influence of the direction and strength of stellar magnetic dipole, as well as the grain size on variability of the spectrum first, and then conclude the paper. 


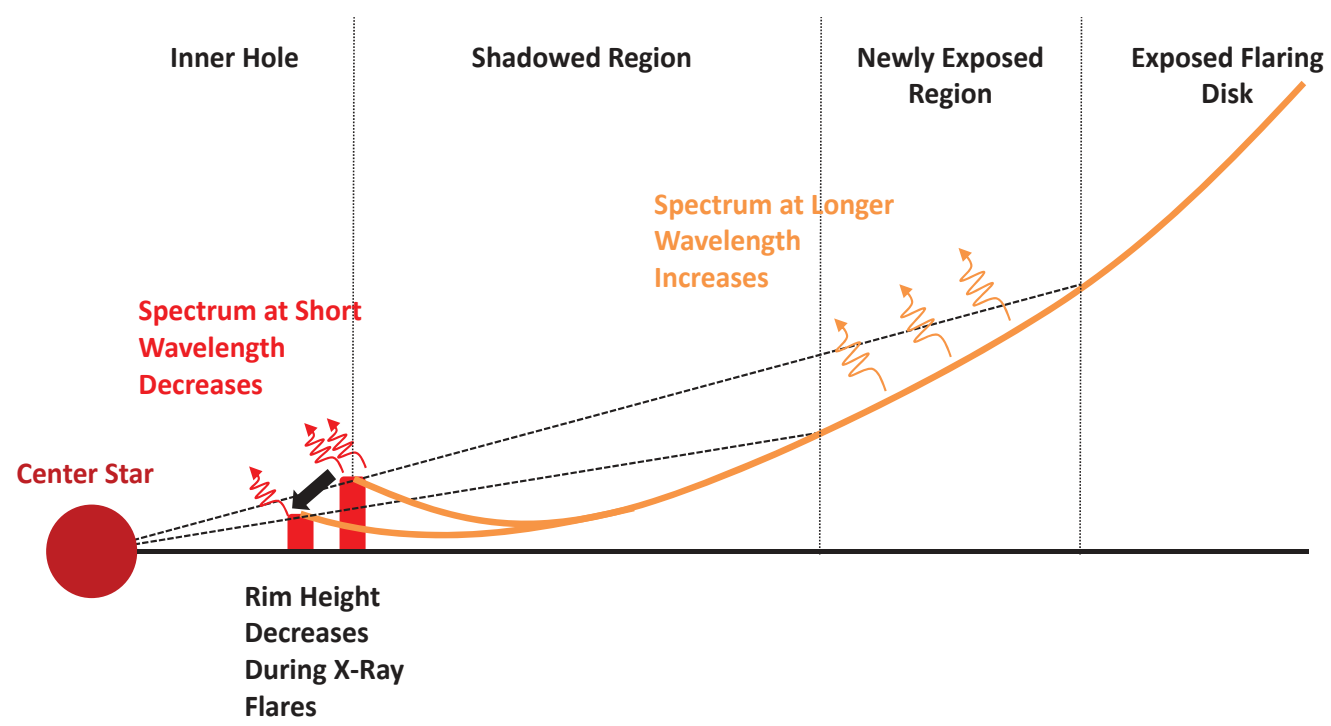

Fig. 1. - A sketch illustrating the spectral variability resulting from disk structural variability during X-ray flares.

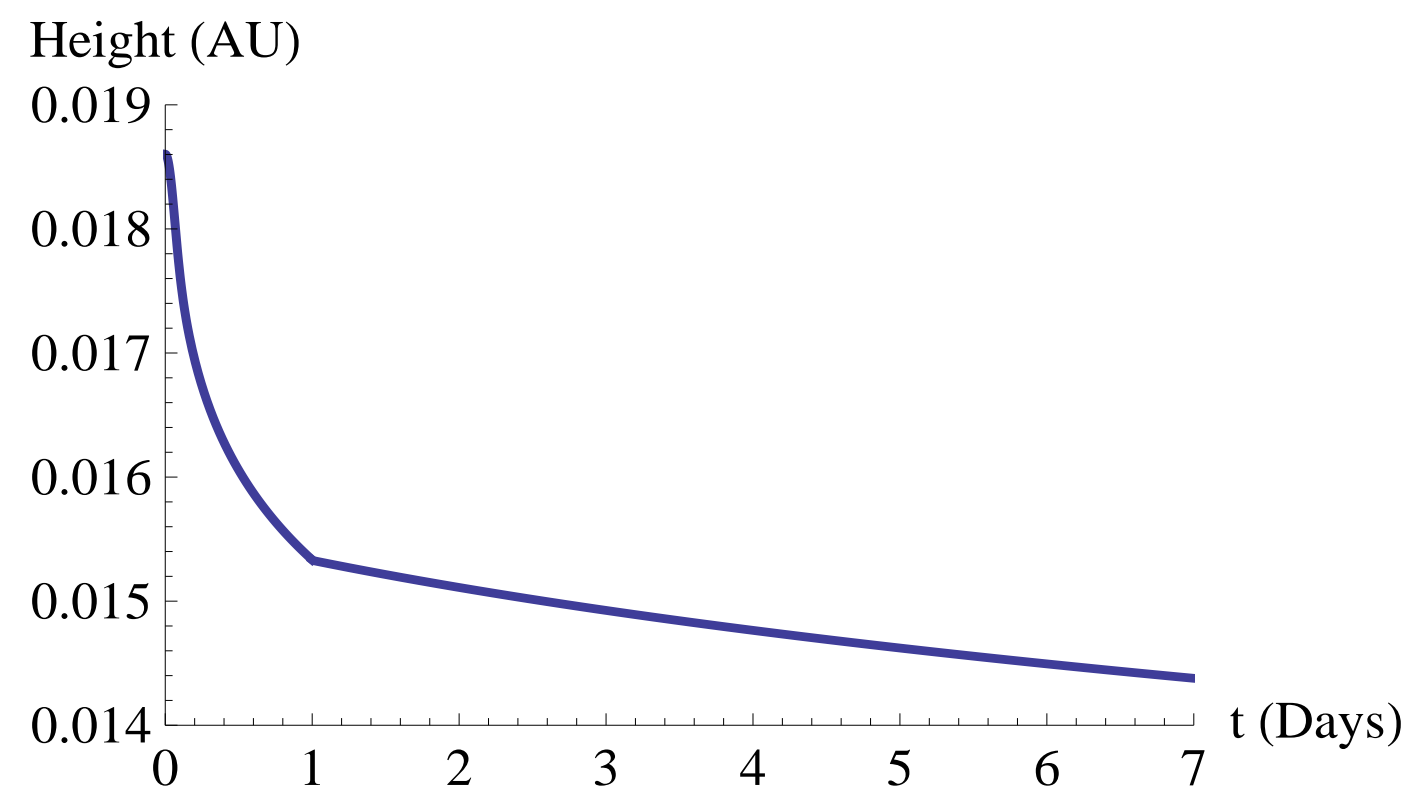

Fig. 2.- Height of inner rim decreases over time, in which case X-ray flare occurs at time $t=0$ day and ends at time $t=1$ day. 


\section{Hrim}

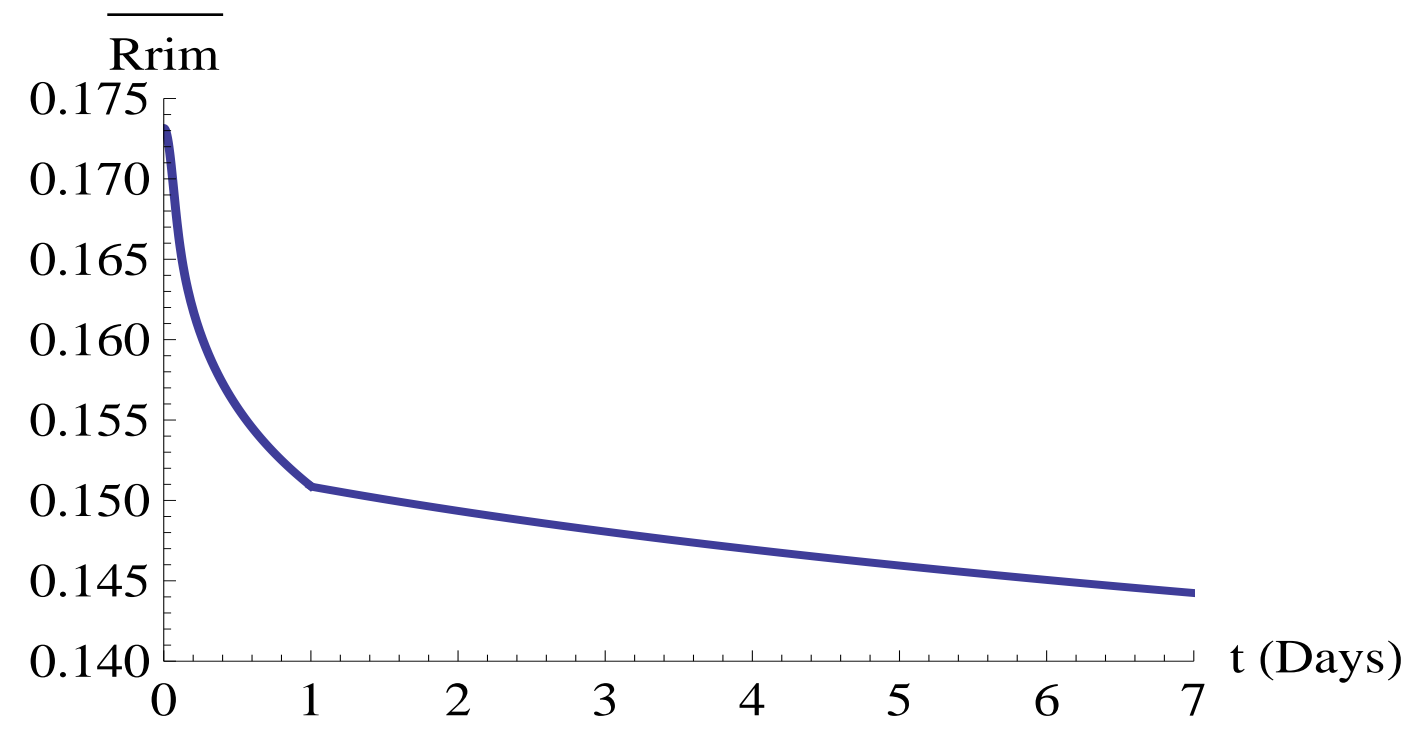

Fig. 3.- Ratio of height over radius of the inner rim decreases over time, in which case X-ray flare occurs at time $t=0$ day and ends at time $t=1$ day.

In our numerical example above, the stellar magnetic moment is parallel with the angular momentum of the disk. However, in a general situation, the angle between the stellar magnetic moment and angular momentum of the disk can range from 0 to $\pi$, as discussed by (Flaherty and Muzerolle 2010). Here we simply show the result for anti-parallel case (with the angle as $\pi$ ). Contrary to the parallel case, grains strongly negatively charged will be driven by the magnetic field to undergo collective migration outward, so the inner rim of the disk gets puffed up. The variability of the shadowed region can be complicated: on one hand, the puffed-up inner rim cast larger shadow on the flaring disk; on the other hand, some stellar light may penetrate the attenuated puffed-up inner rim. As a result, the spectrum at shorter wavelength increases while the spectrum at longer wavelength may experience decreasing first and increasing later. The variability of the height of inner rim is shown in Fig. 6, and the trajectory of charged grains projected in the midplane is shown in Fig. 7. Because the gas gets much dilute outward, charged grains basically spiral out of the system.

To look at the influence of magnetic field strength on variability of the spectrum, we perform the same numerical simulation except for stellar magnetic field with $B_{s}=500 G s$ and $B_{s}=2000 G s$ respectively in the parallel case. We expect stronger magnetic field means stronger Lorentz force, resulting in more variability. In fact, for stellar magnetic field with $B_{s}=500 G s$, the height of inner rim decreases by $15.5 \%$ in the first day, and decreases by $20.6 \%$ cumulatively in seven days after occurrence X-ray flares. Spectrum at $\lambda=5 \mu \mathrm{m}$ decreases by $13.4 \%$ and $17.2 \%$ respectively within one day and the whole week; while spectrum at $\lambda=15 \mu \mathrm{m}$ increases by $6.5 \%$ and $8.5 \%$ respectively within one day and the whole week. The numerical result indicates that weaker magnetic field can 


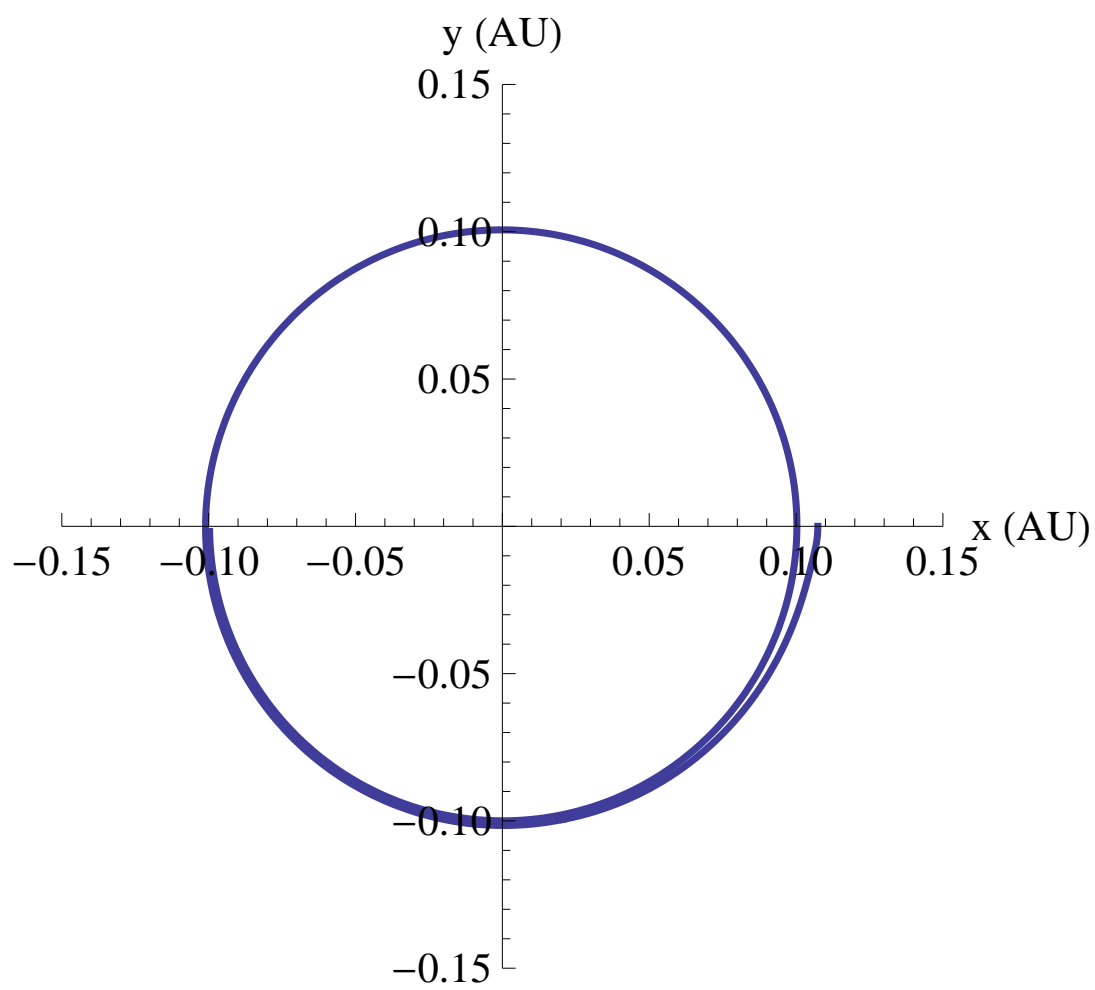

Fig. 4.- Trajectory of charged grains projected in the midplane (in the reference framework corotating with the central star), in which case X-ray flare occurs at time $t=0$ day and ends at time $t=1$ day. 


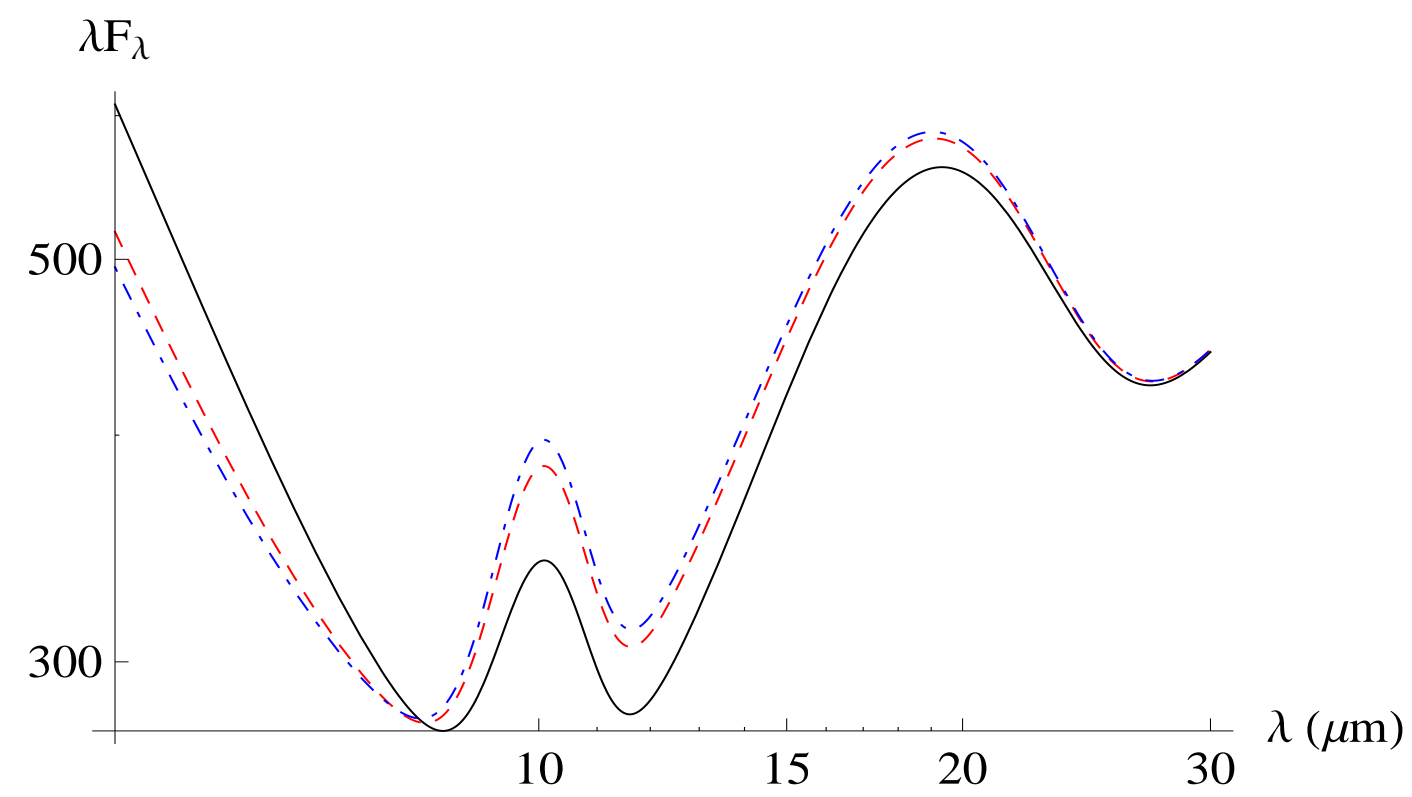

Fig. 5.- Log-log plot of multiple spectra. The solid black line represents the total spectrum of the star-disk system at time $t=0$ day; the dashed red line represents the total spectrum at time $t=1$ day, one day after the occurrence of X-ray flare; and the dot-dashed blue line stands for the spectrum at time $t=7$ day, six days after the cease of X-ray flare. A clear seesaw feature is shown here: the spectrum at shorter wavelength decreases and spectrum at longer wavelength increases over time, with a pivot around $8 \nu \mathrm{m}$. The spectral variability is around $20 \%$ within one week. 


\section{Height (AU)}

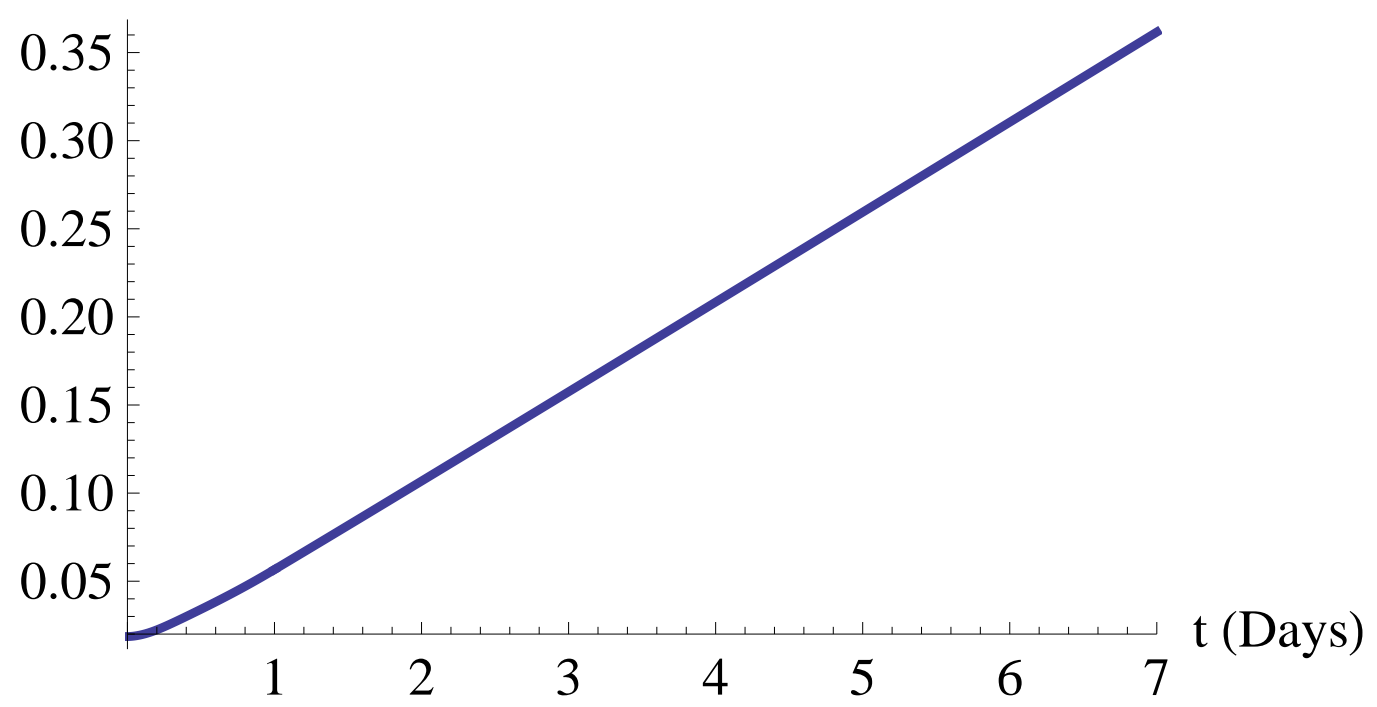

Fig. 6. - In the anti-parallel case, height of inner rim increases over time, in which case X-ray flare occurs at time $t=0$ day and ends at time $t=1$ day.

generate significant variability as well. On contrary for stellar magnetic field with $B_{s}=2000 G s$, the height of inner rim decreases by $19.7 \%$ in the first day, and decreases by $24.9 \%$ cumulatively in seven days after occurrence X-ray flares. Spectrum at $\lambda=5 \mu m$ decreases by $16.4 \%$ and $20.1 \%$ respectively within one day and the whole week; while spectrum at $\lambda=15 \mu \mathrm{m}$ increases by $8.2 \%$ and $9.9 \%$ respectively within one day and the whole week. Spectrum indeed becomes more volatile for stronger stellar magnetic field.

To look at the influence of grain size on variability of the spectrum, we perform the same numerical simulation except that grain radius $r_{d}=0.1 \mu \mathrm{m}$ and $r_{d}=1 \mu \mathrm{m}$ respectively in the parallel case. Mass of grains is proportional to $r_{d}^{3}$, while the Lorentz force is proportional to $Q_{0}$, which is proportional to $r_{d}$, thus smaller grains experience larger force per unit mass, therefore are expected to display more variability. In fact, for grains with radius $r_{d}=0.1 \mu m$, the height of inner rim decreases by $22.5 \%$ in the first day, and decreases by $21.7 \%$ cumulatively in seven days after occurrence X-ray flares. Spectrum at $\lambda=5 \mu \mathrm{m}$ decreases by $21.7 \%$ and $26.7 \%$ respectively within one day and the whole week; while spectrum at $\lambda=15 \mu \mathrm{m}$ remains almost unchanged with decrease by $0.5 \%$ and $1 \%$ respectively within one day and the whole week. The numerical result is consistent with our analysis, and the spectrum also decreases at longer wavelength due to a sharp decrease in the spectrum from the inner rim. On contrary for grains with radius $r_{d}=1 \mu \mathrm{m}$, the height of inner rim decreases by $15.5 \%$ in the first day, and decreases by $20.8 \%$ cumulatively in seven days after occurrence X-ray flares. Spectrum at $\lambda=5 \mu m$ decreases by $11.9 \%$ and $14.8 \%$ respectively within one day and the whole week; while spectrum at $\lambda=15 \mu \mathrm{m}$ increases by $8.1 \%$ and $10.7 \%$ 


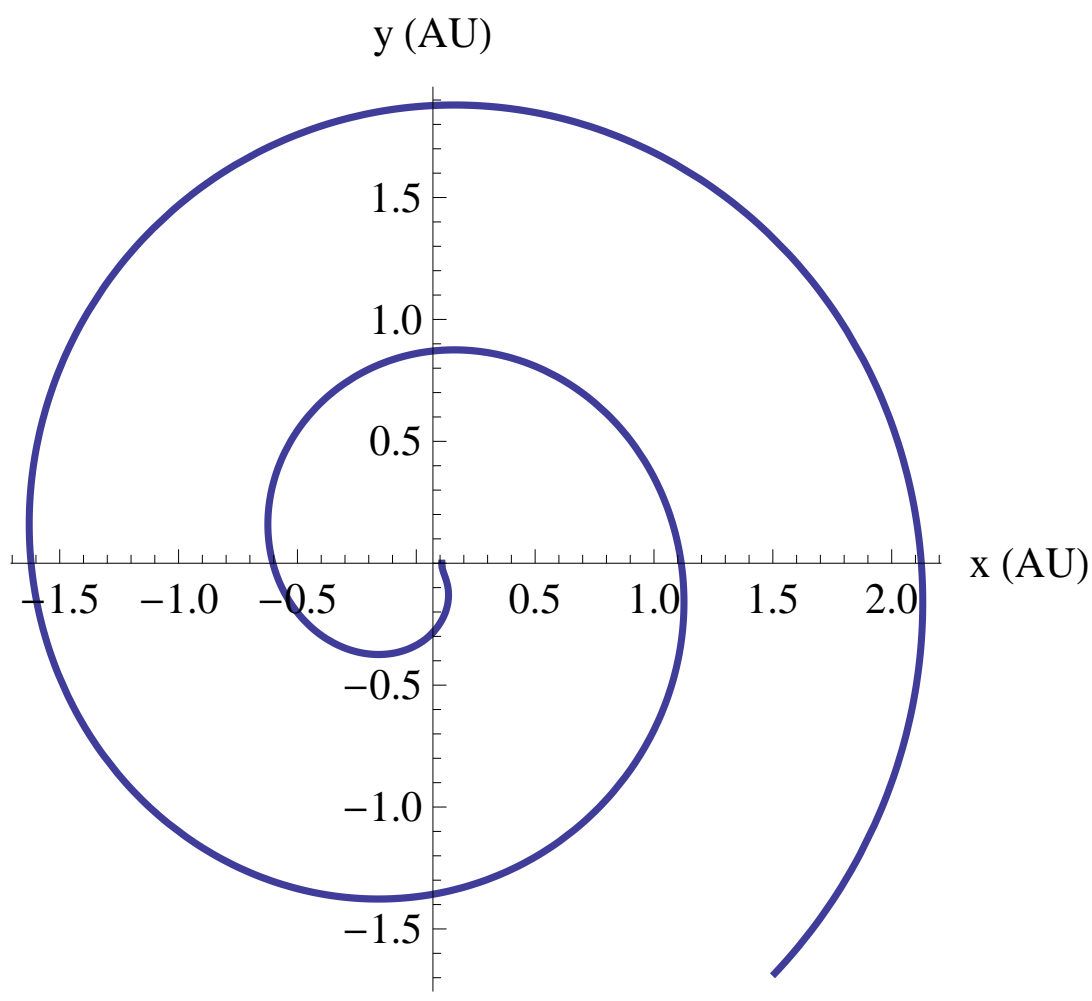

Fig. 7.- Trajectory of charged grains projected in the midplane in the anti-parallel case (in the reference framework corotating with the central star), in which case X-ray flare occurs at time $t=0$ day and ends at time $t=1$ day. 
respectively within one day and the whole week.

In conclusion, we construct a physical model to attribute the puzzling rapid MIR spectral variability to the observed X-ray flares from T Tauri stars. Our simulation result captures all main observed features consistently, and is robust with respect to different grain size and strength of stellar magnetic field. We made various assumptions in the construction of this model, including the location of the $R_{\text {rim }}$ and an assumed distribution of the disk aspect ratio. The latter can be mapped out with regular monitor of disk's SED changes. Both of these quantities may also depend on the stellar magnetic field. It may be useful to explore correlations, if any, between these

observable quantities. Finally, the basic assumption that the SED variations are triggered by X-ray flares may be tested by simultaneous X-ray and infrared observations.

\section{Acknowledgments}

We thank Xu Huang and Wenhua Ju for their constructive suggestions. This work is supported by NASA (NNX07A-L13G, NNX07AI88G, NNX08AL41G, NNX08AM84G), and NSF(AST0908807).

\section{REFERENCES}

Alencar,S.H.P., $\quad$ Teixeira,P.S., Guimaraes,M.M., $\quad$ McGinnis,P.T., Gameiro,J.F., $\quad$ Bouvier,J., Aigrain,S., Flaccomio,E. \& Favata,F., 2010, A\&A, 519, A88

Aly,J.J., 1980, A\&A, 86, 192-197

Aarseth,S.J., 2003, Gravitational N-Body Simulations, Cambridge University Press, Cambridge

Bary,J.S., Leisenring,J.M. \& Skrutskie,M.F., 2009, ApJ, 706, L168-L172

Chiang,E.I. \& Goldreich,P., 1997, ApJ, 490, 368

Crosby,N.B., Aschwanden,M.J., \& Dennis,B.R. 1993, Sol. Phys., 143, 275

Dalgarno, A., \& McCray,R.A. 1972, ARA\&A, 10, 375

Draine, B.T. 2010, Physics of the Interstellar and Intergalactic Medium (Princeton, NJ: Princeton Univ. Press)

Dullemond,C.P., Dominik,C. \& Natta,A., 2001, ApJ, 560, 975D

Dullemond,C.P., \& Monnier,J.D., 2010, Annu. Rev. A\&A, 48, 205-39

Espaillat,C.,Furlan,E., DAlessio,P., Sargent,B., Nagel,E., Calvet,N., Watson,D.M. \& Muzerolle,J., 2011, ApJ, 728, 49 
Favata,F., Flaccomio,E., Reale,F., Micela,G., Sciortino,S., Shang,H., Stassun,K.G. \& Feigelson,E.D., 2005, ApJ, 160, 469-502

Feigelson,E.D. \& Montmerle,T., 1999, Annu. Rev. Astron. Astrophys, 37, 363-408

Flaherty,K.M., Muzerolle,J., Rieke,G., Gutermuth,R., Balog,Z., Herbst,W., Megeath,S.T. \& Kun,M., 2011, ApJ, 732, 83

Flaherty,K.M. \& Muzerolle,J., 2010, ApJ, 719, 1733-1749

Garaud,P. \& Lin,D.N.C., 2006, ApJ, 654, 606

Getman,K.V., Feigelson,E.D., Broos,P.S., Micela,G., Garmire,G.P., 2008, ApJ, 688, 418-436

Ghosh,P. \& Lamb,F.K., 1979, ApJ, 232, 259-276

Ghosh,P. \& Lamb,F.K., 1979, ApJ, 234, 296-316

Giardino,G., Favata,F., Pillitteri,I., Flaccomio,E., Micela,G., \& Sciortino,S. 2007, A\&A , 475, 891

Glassgold,A.E., Najita,J.\& Igea,J., 1997, ApJ, 480, 344

Glassgold,A.E., Feigelson,E.D., Momtmerle,T.\& Wolk,S., 2005, ApJ, 341,165G

Gregory,S.G., Wood,B.K., \& Jardine,M., 2007, MNRAS, 379, L35-L39

Gudel,M. \& Telleschi,A., 2007, A\&A, 474, L25-L28

Hayashi,M.R., Shibata,K. \& Matsumoto,R., 1996, ApJ, 468, L37-L40

Hudson,H.S. 1991, Sol. Phys., 133, 357

Jardine,M., Gregory,S.G. \& Donati,J.F., 2007, Proceedings IAU Symposium, No. 243

Johns-Krull,C.M., Valenti,J.A. \& Saar,S.H., 2004, ApJ, 617, 1204-1215

Johns-Krull,C.M., 2007, ApJ, 664, 975-985

Konigl,A., 1991, ApJ, 370, L39-L43

Lai,D., 1999, ApJ, 524, 1030-1047

Lin,D.N.C. \& Murray,S.D., 1992, ApJ, 394, 523-533

Livio,M. \& Pringle,J.E., 1992, MNRAS, 259, 23p-26p

Murray,S.D. \& Lin,D.N.C., 1992, ApJ, 384, 177-184

Muzerolle,J., Flaherty,K., Balog,Z., Furlan,E., Smith,P.S., Allen,L., Calvet,N., DAlessio,P., Megeath,S.T., Muench,A., Rieke,G.H. \& Sherry,W.H., 2009, ApJ, 704, L15-L19 
Osterbrock,D.E. \& Ferland, G.J. 2006, Astrophysics of gaseous nebulae and active galactic nuclei (Mill Valley, CA: University Science Books)

Pringle,J.E., \& Rees,M.J., 1972, A\&A, 21, 1

Reale,F., Betta,R., Peres,G., Serio,S., \& McTiernan,J. 1997, A\&A, 325, 782

Ruden,S.P \& Lin,D.N.C., 1986, ApJ, 308, 883

Shakura,N.I \& Sunyaev,R.A., 1973, Astr.Ap., 24, 337

Shu,F., Najita,J., Ostriker,E., Wilkin,F., Ruden,S. \& Lizano,S., 1994, ApJ, 429, 781-796

Sitko,M.L et al., 2008, ApJ, 678, 1070-1087

Stelzer,B., et al. 2005, ApJS, 160, 55

Umebayashi,T., 1983, Progress of Theoretical Physics, Vol.69, No.2, Feb

Weingartner,J.C., Draine,B.T. \& Barr, D.K., 2006, ApJ, 645, 1188-1197

Wolk,S.J., Harnden Jr,F.R., Flaccomio,E., Micela,G., Favata,F., Shang,H., Feigelson,E.D., ApJ, 2005, 160, 423-449 